Sedelbankstatistik

Author(s): Ivar Hultman

Source: Ekonomisk Tidskrift, Årg. 15, häft 5 (1913), pp. 180-199

Published by: Wiley on behalf of The Scandinavian Journal of Economics

Stable URL: http://www.jstor.org/stable/3437248

Accessed: 27-06-2016 15:55 UTC

Your use of the JSTOR archive indicates your acceptance of the Terms \& Conditions of Use, available at

http://about.jstor.org/terms

JSTOR is a not-for-profit service that helps scholars, researchers, and students discover, use, and build upon a wide range of content in a trusted digital archive. We use information technology and tools to increase productivity and facilitate new forms of scholarship. For more information about JSTOR, please contact support@jstor.org.

Wiley, The Scandinavian Journal of Economics are collaborating with JSTOR to digitize, preserve and extend access to Ekonomisk Tidskrift 


\title{
Sedelbankstatistik.
}

\author{
Af
}

Ivar Hultman.

Hvar och en, som har anledning att något mera ingående syssla med de olika sedelbankernas verksamhet, torde känna och tillfullo uppskatta det synnerligen värdefulla jämförelsematerial, som föreligger $\mathrm{i}$ den af tyska riksbankens statistiska afdelning till I908 års bankenquête utarbetade „Vergleichende Notenbankstatistik».

Som emellertid nu fem år förflutit sedan denna statistik publicerades, och materialet sålunda väsentligt förlorat $\mathrm{i}$ aktualitet, har jag trott att följande öfversikter rörande åren I908-I9 I 2 skulle vara af ett visst värde för dem som närmare intressera sig för bankstatistiken. Jag har då medtagit åtskilliga flere banker än som i den ofvannämnda statistiken blifvit behandlade, så att, under det den senare omfattar endast 7 banker, här lämnas öfversikter rörande ej mindre än $\mathrm{I} 8$ banker, af hvilka dock några $\mathrm{i}$ brist på primärmaterial blifvit relativt ofullständigt behandlade. Af samtliga europeiska centralbanker äro endast tre helt utelämnade, nämligen Greklands, Montenegros och Turkiets.

Ett försök till likformig behandling af ett så stort antal banker måste helt naturligt stöta på afsevärda svårigheter på grund af rapporternas olikformiga uppställning. Särskildt är omfattningen af andra avistaförbindelser än sedelcirkulationen svår att afgränsa utan biträde från de beträffande institutionerna själfva, och beträffande en eller annan bank kunna nog $\mathrm{i}$ det afseendet misstag vara begångna. Vidare angifva vissa banker i sina årsberättelser medeltal, som tydligen grunda sig på annat eller ytterligare material än de publicerade veckorapporterna. Och slutligen äro understundom vissa betydelsefulla förhållanden undandragna publikation. 
Men jag hyser den - kanske något förmätna - förhoppningen att så småningom kunna åstadkomma ett fullt jämförbart material i den mån de beträffande bankerna själfva kunna intresseras för saken, och jag anser mig kunna göra detta på grund af det stora tillmötesgående jag i mitt studium af de europeiska centralbankerna redan rönt från dessas sida. Det senaste beviset på tillmötesgående är Nationalbankens välvilligt lämnade specifikation af sin smetallfonds» sammansättning vid hvarje månads utgång, en specifikation, som hittills aldrig blifvit publicerad.

De $\mathrm{i}$ öfversikterna angifna siffrorna afse medeltal för kalenderår utom beträffande sedelcirkulationens uppdelning på olika valörer, hvilken på ett undantag när afser visst datum på året.

\begin{tabular}{|c|c|c|c|c|c|}
\hline \multicolumn{6}{|c|}{$\begin{array}{c}\text { Bank of England } \\
\text { (i IOoO-tal } f)^{1}\end{array}$} \\
\hline & 1908 & 1909 & I9IO & I9I I & 1912 \\
\hline Guld . . . . . . . & 36,457 & 36,615 & 36,142 & 37,363 & 37,938 \\
\hline Silfver $^{2} \ldots \ldots$ & 800 & 800 & 800 & 798 & 800 \\
\hline Summa metall . . . . . & 37,257 & 37,415 & 36,942 & 38,161 & 38,738 \\
\hline Sedelcirkulation . . . . & 28,857 & 29,215 & 28,255 & 28,609 & 28,790 \\
\hline Sedelreserv . . . . • & 26,850 & 26,650 & $27, \mathrm{I} 37$ & 28,002 & 28,398 \\
\hline Sedelcirk. täckn. af metall . . & $\mathrm{I} 29, \mathrm{II} \%$ & I $28,07 \%$ & I $30,75 \%$ & I $33,39 \%$ & I $34,55 \%$ \\
\hline$\bowtie \quad »$ guld.. & I $26,34 \%$ & $125,33 \%$ & $\mathrm{I} 27,9 \mathrm{I} \%$ & $130,60 \%$ & $\mathrm{I} 3 \mathrm{I}, 78 \%$ \\
\hline Avistaförbindelser till staten . & 9,214 & I0,519 & I 3,078 & I 3,088 & I 7,477 \\
\hline Öfriga avistaförbindelser... & 44,008 & 43,795 & $4 \mathrm{I}, 795$ & 42,396 & 42,129 \\
\hline Summa avistaförbindelser . . . & 82,079 & 83,529 & $83, \mathbf{1} 28$ & 84,093 & 88,396 \\
\hline Avistaförb. täckn. af metall . . & $45,39 \%$ & $44,79 \%$ & $44,44 \%$ & $45,38 \%$ & $43,82 \%$ \\
\hline$\bowtie \quad \gg$ guld. & $44,42 \%$ & $43,84 \%$ & $43,48 \%$ & $44,43 \%$ & $42,92 \%$ \\
\hline \multicolumn{6}{|l|}{ Governm. securities in Banking } \\
\hline Department . . . . & 14,857 & I 5,457 & I 5,487 & 14,872 & I3,977 \\
\hline Other securities in Bank. Dept. & 29,428 & 30,082 & 30,160 & 30,514 & $35, \mathrm{r} 9 \mathrm{I}$ \\
\hline Växeldiskonto . . . • . & $3,010 \%$ & 3, IOI $\%$ & $3,717 \%$ & $3,469 \%$ & $3,774 \%$ \\
\hline
\end{tabular}




\section{Banque de France}

\begin{tabular}{|c|c|c|c|c|c|}
\hline & 1908 & 1909 & I910 & I9I I & 1912 \\
\hline Guld $^{1}$. . . . . . . & $3,052,000$ & $3,630,400$ & $3,400,000$ & $3,205,700$ & $3,239,000$ \\
\hline$\cdot \cdot \cdot \cdot \cdot$ & 904,700 & 893,800 & 861,600 & 830,700 & 788,900 \\
\hline Summa metall . . . . . & $3,956,700$ & $4,524,200$ & $4,261,600$ & $4,036,400$ & $4,027,900$ \\
\hline Sedelcirkulationen $^{1}$. . . & $4,853,44 \mathrm{I}$ & $5,079,925$ & $5,197,762$ & $5,243,375$ & $5,322,937$ \\
\hline \multicolumn{6}{|l|}{ Sedelcirkulationens } \\
\hline täckn. af metall ..... & $81,52 \%$ & $89,06 \%$ & $8 \mathrm{I}, 99 \%$ & $76,98 \%$ & $75,67 \%$ \\
\hline$» \quad$ guld . . . . & $62,88 \%$ & $71,47 \%$ & $65,4 \mathrm{I} \%$ & $61, \mathbf{1} \mathbf{4} \%$ & $60,85 \%$ \\
\hline Avistaförbindelser till staten ${ }^{23}$ & 170,07 I & I $85,08 \mathrm{I}$ & I 31,879 & 200,317 & 246,691 \\
\hline Öfriga avistaförbindelser ${ }^{24} \cdot$. & 559,344 & 705,446 & $647, \mathrm{I} 33$ & 628,802 & 705,206 \\
\hline Summa avistaförbindelser. . . & $5,582,856$ & $5,970,452$ & $5,976,774$ & $6,072,494$ & $6,274,834$ \\
\hline \multicolumn{6}{|l|}{ Avistaförbindelsernas } \\
\hline täckn. af metall . . & $70,87 \%$ & $75,78 \%$ & $71,30 \%$ & $66,47 \%$ & $64,19 \%$ \\
\hline$» \quad$ guld ..... & $54,67 \%$ & $60,8 \mathrm{r} \%$ & $56,89 \%$ & $52,79 \%$ & $51,62 \%$ \\
\hline Växelportfölj ${ }^{2} \ldots \ldots$ & 897,200 & $76 \mathrm{I}, 500$ & 977,300 & $\mathrm{I}, 203,700$ & $\mathrm{I}, 332,700$ \\
\hline Lombardlån $^{25}$. . . . . & 527,800 & 513,600 & 549,900 & 638,500 & 684,800 \\
\hline Växeldiskonto $^{1} \cdot \ldots \cdot \cdot \cdot$ & $3,04 \%$ & $3,00 \%$ & $3,00 \%$ & $3, \mathrm{x} 3 \%$ & $3,37 \%$ \\
\hline Sedelvalörer $^{1}$. . . . . . & $28 / 1-09$ & $23 / 12-09$ & $22 / 12-10$ & $2 \mathrm{I} / \mathrm{I} 2-\mathrm{II}$ & $19 / 12-12$ \\
\hline I,000 . . . . . & $\mathbf{I}, 366,948$ & $\mathrm{I}, 43^{8,227}$ & $\mathrm{I}, 454,554$ & I,466,446 & $\mathrm{I}, 545,4 \mathrm{I} 7$ \\
\hline $500 \cdot \cdot \cdot \cdot \cdot \cdot \cdot$ & 293,709 & 295,601 & 289,329 & 282,850 & 288,949 \\
\hline 100........ & $2,636,02 \mathrm{I}$ & $2,698, \mathrm{IO} 5$ & $2,756,6$ Io & $2,779,903$ & $2,906,437$ \\
\hline $50 \cdot \cdot \cdot \cdot \cdot \cdot \cdot$ & 694,983 & 705,228 & 649,196 & 694,265 & 799,140 \\
\hline $25 \cdot \cdot \cdot \cdot \cdot \cdot \cdot \cdot$ & 384 & 383 & 382 & $38 \mathrm{I}$ & 380 \\
\hline $20 \cdot \cdot \cdot \cdot \cdot \cdot \cdot \cdot$ & $\mathrm{I}, 25 \mathrm{I}$ & I, 243 & I, 236 & $\mathrm{I}, 227$ & $\mathrm{I}, 22 \mathrm{I}$ \\
\hline \multirow[t]{2}{*}{$5 \cdot \ldots \cdot \ldots \cdot \cdot$} & 680 & 678 & 677 & 675 & 674 \\
\hline & $4,993,976$ & $5, \mathrm{I} 39,465$ & $5, \mathrm{I} 5 \mathrm{I}, 984$ & $5,225,747$ & $5,542,2 \mathrm{I} 8$ \\
\hline
\end{tabular}

1 Enligt bankens årsberättelser.

2 Uträkningar enligt veckouppgifterna.

3 Compte courant du Trésor.

4 Comptes courants et dépôts de fonds des particuliers, billets à ordre et récépissés à vue samt arrérages de valeurs transférées ou déposées.

${ }^{5}$ Avances sur titres. 


\section{Reichsbank}

(i I,000-tal Rmk).

\begin{tabular}{|c|c|c|c|c|c|}
\hline & I908 & 1909 & I910 & I9I I & I9I 2 \\
\hline Guld $^{1}$. . . . . . . & 785,195 & $795,25 \mathrm{I}$ & 777,834 & 827,624 & 880,083 \\
\hline Skiljemynt ${ }^{1}$ & 233,870 & 251,082 & 277,969 & 301,6 I 8 & 323,512 \\
\hline Summa metall ${ }^{1}$. & I,019,065 & I,046,333 & $1,055,803$ & $\mathrm{I}, \mathrm{1} 29,242$ & I, 203,595 \\
\hline 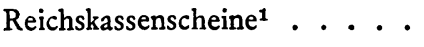 & $67, \mathrm{II} 4$ & 67,280 & 64,288 & 55,748 & 39, I 18 \\
\hline Summa "Barvorrat» .... & I,086,I 79 & I 1 I 3,6 I 3 & $1,120,091$ & I,I 84,990 & $1,242,7 \mathrm{I} 3$ \\
\hline Sedelcirkulationen & $\mathrm{I}, 524, \mathrm{I} 42$ & $1,576,532$ & $1,605,882$ & I,663,6I 5 & I,781,999 \\
\hline \multicolumn{6}{|l|}{ Sedelcirkulationens } \\
\hline täckn. af Barvorrat .. & $71,26 \%$ & $70,64 \%$ & $69,75 \%$ & $71,23 \%$ & $69,74 \%$ \\
\hline » metall .... & $66,86 »$ & $66,37 »$ & $65,74 »$ & $67,88 \gtrsim$ & $67,54 »$ \\
\hline$» \quad$ guld & $51,52 》$ & $50,44 »$ & $48,44 》$ & $49,75 》$ & $49,39 \gg$ \\
\hline Öfriga avistaförbindelser ${ }^{1} \cdot \cdot$ & $649,29 \mathrm{I}$ & 722,370 & 648,704 & 653,174 & 718,197 \\
\hline Summa avistaförbindelser . . & $2,173,433$ & $2,298,902$ & $2,254,586$ & $2,316,789$ & 2,500,196 \\
\hline \multicolumn{6}{|l|}{ Avistaförbindelsernas } \\
\hline täckning af metall $\ldots$. & $46,89 \%$ & $45,51 \%$ & $46,83 \%$ & $48,74 \%$ & $48,14 \%$ \\
\hline$\bowtie$ guld $\ldots$. & 36,13 & 34,59 & $34,50 \gtrsim$ & $35,72 »$ & $35,20 »$ \\
\hline Växelportföljen ${ }^{1}$ & 967,729 & 918,894 & 994,446 & $\mathrm{I}, 077,777$ & $\mathrm{I}, 238,325$ \\
\hline Lombardlån ${ }^{1}$. . & 91,397 & 87,591 & 98,443 & 78,958 & 82,430 \\
\hline Växeldiskonto . . . • • & $4,76 \%$ & $3,93 \%$ & $4,35 \%$ & $4,40 \%$ & $4,95 \%$ \\
\hline Skattefri reserv $^{2} \ldots \ldots$. & $57,5 \mathrm{ro}$ & 31,865 & IO, 575 & I I 3,277 & 54,680 \\
\hline \multicolumn{6}{|l|}{ Sedelvalörer ${ }^{1}$ den 31 december } \\
\hline$\cdots \cdot \cdot \cdot$ & 383,560 & 387,624 & 370,952 & 375,287 & 390,959 \\
\hline$\cdot \cdot \cdot \cdot$ & $1,308,680$ & $1,401,723$ & $\mathrm{I}, 427,237$ & $I, 508,268$ & I,589,466 \\
\hline$\cdots \cdot \cdot$ & I 37,229 & 141,354 & 142,134 & I 70,667 & 215,718 \\
\hline \multirow[t]{2}{*}{$20 \cdot \cdot \cdot \cdot \cdot \cdot \cdot \cdot$} & 145,921 & $\mathrm{I} 4 \mathrm{O}, 8 \mathrm{II}$ & I 32,443 & 196,342 & 323,235 \\
\hline & $\mathrm{I}, 975,390$ & $2,07 \mathrm{I}, 5 \mathrm{I} 2$ & $2,072,766$ & $2,250,564$ & $2,519,578$ \\
\hline
\end{tabular}

1 Enligt bankens årsberättelser.

2 Uträkningar enligt veckorapporterna. 


\section{Österreichisch-ungarische Bank}

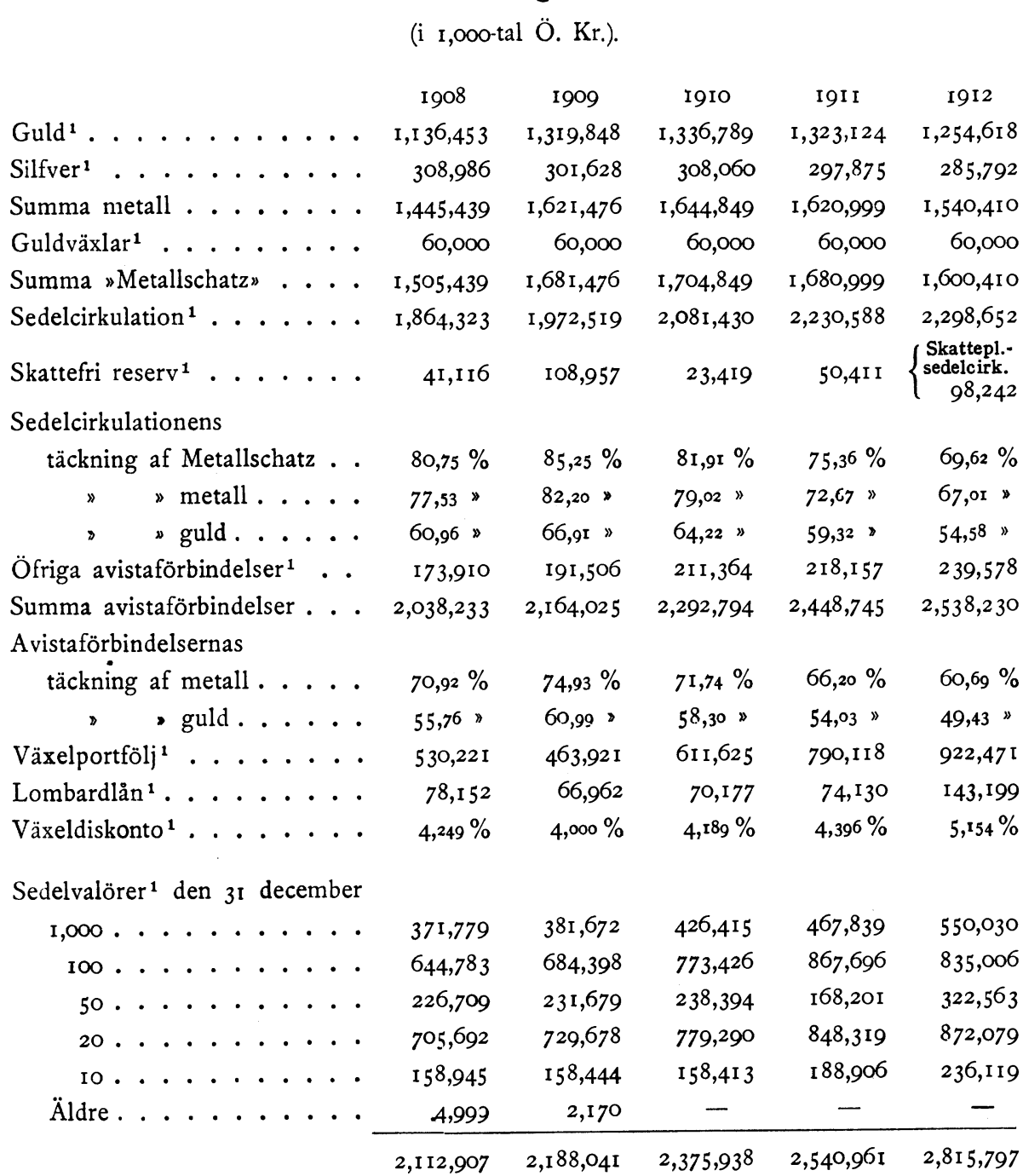

1 Enligt bankens årsberättelser. 


\section{Banca d'Italia}

(i I,000-tal lire).

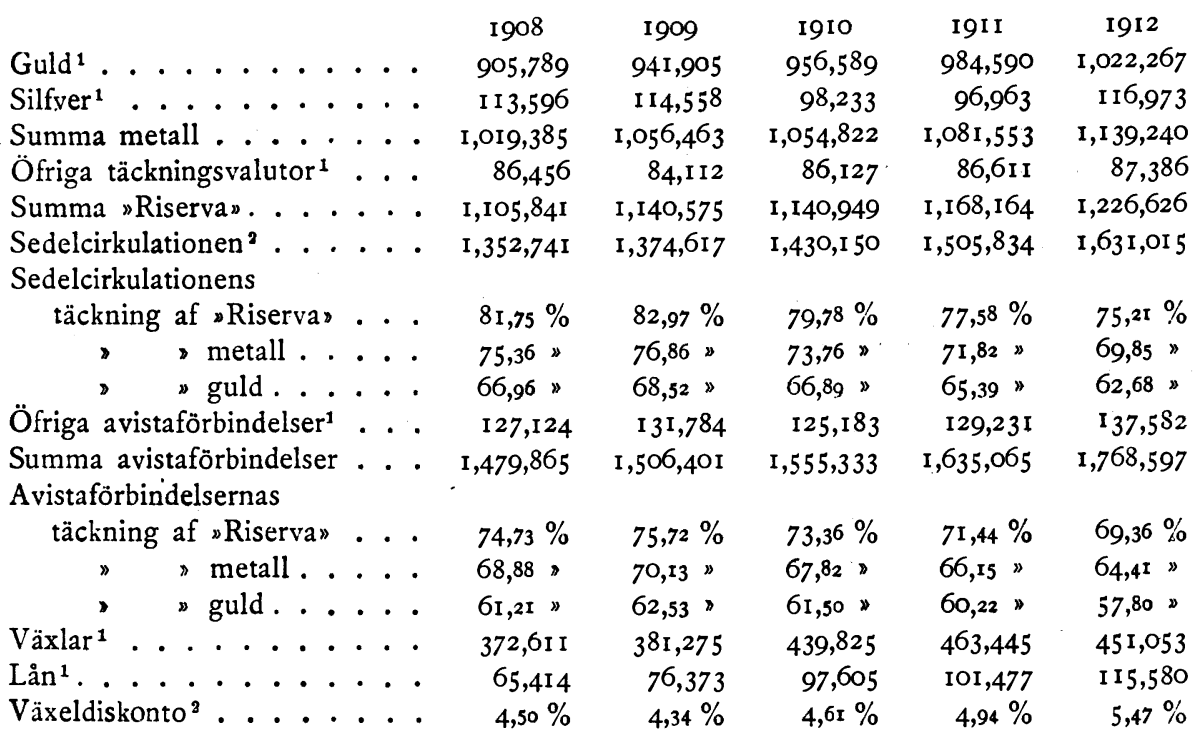

\section{Ryska riksbanken}

(i I,000-tal rubel). ${ }^{3}$

\begin{tabular}{|c|c|c|c|c|c|}
\hline & 1908 & 1909 & I9IO & I9I I & I912 \\
\hline Guld . . . . . . . & I,OI 2,310 & I, I27,040 & $\mathrm{I}, 2 \mathrm{I} 4,043$ & $1,258,245$ & I,294,902 \\
\hline Silfver. & $70,03 \mathrm{I}$ & 79,469 & 76,628 & 69,607 & 72,128 \\
\hline Summa metall ...... & $\mathrm{I}, 082,34 \mathrm{I}$ & I,206,509 & $I, 290,67 \mathrm{I}$ & $1,327,852$ & $1,367,030$ \\
\hline Utländska tillgodohafvanden. . & I 57,031 & I6 $4,42 \mathrm{I}$ & 212,835 & 195,800 & 225,166 \\
\hline Summa kassa. . . . . & $1,239,372$ & $1,370,930$ & $\mathrm{I}, 503,506$ & $1,523,652$ & I, 592,196 \\
\hline Sedelcirkulation. . . . . & $1,093,366$ & $\mathrm{I}, \mathrm{I} 23,984$ & $I, 178,55^{2}$ & $1,263,586$ & $\mathrm{I}, 375,784$ \\
\hline edelcirkulationens & & & & & \\
\hline täckning af kassa : & I I $3,35 \%$ & I 2 I,97 \% & $127,57 \%$ & I $20,58 \%$ & II $5,73 \%$ \\
\hline " metall : & $98,99 *$ & 107,34 & 109,51 & $105,09 »$ & $99,36 »$ \\
\hline guld. . . . . . & 92,59 》 & $100,27 *$ & IO3,or & $99,58 »$ & $94, \mathrm{x} 2 \#$ \\
\hline Avistaförbindelser till staten. . & I 3,920 & 169,622 & 237,693 & 432,783 & 473,570 \\
\hline Öfriga avistaförbindelser . . • & 483,990 & 495,836 & 527,655 & 520,275 & 561,098 \\
\hline $\begin{array}{l}\text { Summa avistaförbindelser . } \\
\text { Avistaförbindelsernas }\end{array}$ & I,69I, 276 & I, $789,44^{2}$ & $\mathrm{I}, 943,900$ & $2,216,644$ & $2,410,452$ \\
\hline täckning af metall & $64,00 \%$ & $67,42 \%$ & $66,40 \%$ & $59,90 \%$ & $56,7 x \%$ \\
\hline , guld & 59,85 & 62,98 " & $62,45 "$ & 56,76 & 53,72 \\
\hline Ple & 447,049 & 427,803 & $45^{8,175}$ & 659,557 & 760,951 \\
\hline ixeldiskonto $\ldots \ldots$ & $5,95 \%$ & $4,99 \%$ & $4,50 \%$ & $4,50 \%$ & $5, \infty \%$ \\
\hline
\end{tabular}

1 Uträkningar enligt Io-dagars rapporterna.

2 Enligt bankens årsberättelser.

- Uträkningar enligt veckorapporterna i "L'économiste . 


\section{Finlands Bank}

(i I,, $00 \cdot$ tal Fmk).

\begin{tabular}{|c|c|c|c|c|c|}
\hline & 1908 & 1909 & I9IO & I9I I & I9J 2 \\
\hline Guld $^{1} \cdot \ldots \cdot \cdot \ldots$ & 23,800 & 24,177 & 23,927 & 28,895 & 30,454 \\
\hline Silfver $^{1} \ldots \ldots$ & 2,145 & 2,698 & 2,679 & 2,587 & 2,248 \\
\hline Summa metall . . . . . & 25,946 & 26,875 & 26,606 & $3 \mathrm{I}, 482$ & 32,702 \\
\hline 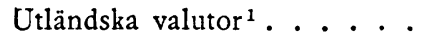 & $8 \mathrm{I}, 7 \mathrm{I2}$ & 83,733 & 107,469 & I 38,004 & 97,954 \\
\hline Summa kassa . . . . . & I07,658 & I Io,608 & I 34,075 & 169,486 & I 30,656 \\
\hline Sedelcirkulation $^{1} \ldots \ldots$ & 92,567 & 90,102 & 120,073 & I 37,990 & I I 5,228 \\
\hline \multicolumn{6}{|l|}{ Sedelcirkulationens } \\
\hline täckning af kassa . . . & I $16,30 \%$ & $122,76 \%$ & I I I, $66 \%$ & I $22,82 \%$ & I I $3,39 \%$ \\
\hline$" \quad "$ metall .... & $28,03 »$ & $29,83 》$ & 22,16, & $22,8 \mathrm{x} »$ & $28,38 》$ \\
\hline$\triangleright \quad \triangleright$ guld . . . . & $25,7 \times 》$ & $26,83 \gg$ & I9,93 》 & $20,94 »$ & $26,43 *$ \\
\hline Öfriga avistaförbindelser ${ }^{1} \cdot \ldots$ & 26,021 & 19,367 & I 8,355 & 29,6 I 7 & 27,329 \\
\hline Summa avistaförbindelser. . . & I 18,588 & 109,469 & I 38,428 & I67,607 & $\mathrm{I} 42,557$ \\
\hline \multicolumn{6}{|l|}{ Avistaförbindelsernas } \\
\hline täckning af kassa .... & $90,78 \%$ & IOI,04 \% & $96,86 \%$ & IOI,12 \% & $9 \mathrm{I}, 65 \%$ \\
\hline $\quad$ metall ... . & $2 \mathrm{I}, 88 》$ & $24,55 »$ & $19,22 》$ & I $8,78 »$ & $22,94 »$ \\
\hline$\triangleright \quad "$ guld $\ldots .$. & $20,07 »$ & $22,09>$ & $17,28 》$ & $17,24 »$ & $2 \mathrm{I}, 36 »$ \\
\hline Obegagnad sedelutgifningsrätt ${ }^{1}$ & 27,128 & 38,603 & 33,457 & $39,53 \mathrm{I}$ & 26,245 \\
\hline 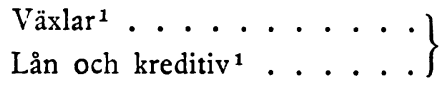 & 81,596 & $7 \mathrm{I}, 2 \mathrm{I} 7$ & 80,716 & 82,916 & $\begin{array}{l}68,756 \\
34,366\end{array}$ \\
\hline Växeldiskonto . . . . • . & $6,16 \mathrm{r} \%$ & $5,158 \%$ & $5,021 \%$ & $4,756 \%$ & $5,104 \%$ \\
\hline
\end{tabular}

Sedelvalörer ${ }^{2}$ den 3 I dec

\begin{tabular}{|c|c|c|c|c|c|}
\hline $\mathrm{I}, 000 \ldots$ & - & 一 & - & I I, 295 & 19,772 \\
\hline $500 \ldots . \ldots$ & I 5,059 & 38,847 & 47,327 & 21,783 & IO,9OI \\
\hline $100 \cdot \cdot \cdot \cdot \cdot$ & 21,789 & $22,06 \mathrm{I}$ & 22,458 & 24,435 & 26,168 \\
\hline $50 \cdot \cdot \cdot \cdot \cdot \cdot \cdot \cdot$ & $7,56_{3}$ & 7,475 & 8,049 & 8,370 & 9,259 \\
\hline $20 . \cdot$ & 16,919 & I7,838 & I 7,484 & 21,004 & 21,770 \\
\hline го........ & I 3,708 & $\mathrm{I} 4, \mathrm{I} 44$ & I6,295 & 16,374 & 17,302 \\
\hline $5 \ldots \ldots$ & 10,398 & I0,709 & I I, 746 & I I 4449 & I I, 777 \\
\hline Äldre valörer. . . & 346 & 346 & 346 & 345 & 345 \\
\hline \multirow[t]{2}{*}{ Rubel } & 207 & 205 & 204 & 204 & 204 \\
\hline & 85,989 & I I I, 625 & I2 3,909 & I 5,259 & I I 7,498 \\
\hline
\end{tabular}

1 Uträkningar enligt månadsrapporterna, I912 enligt veckorapporterna.

2 Enligt bankens årsberättelser. 


\section{Sveriges Riksbank}

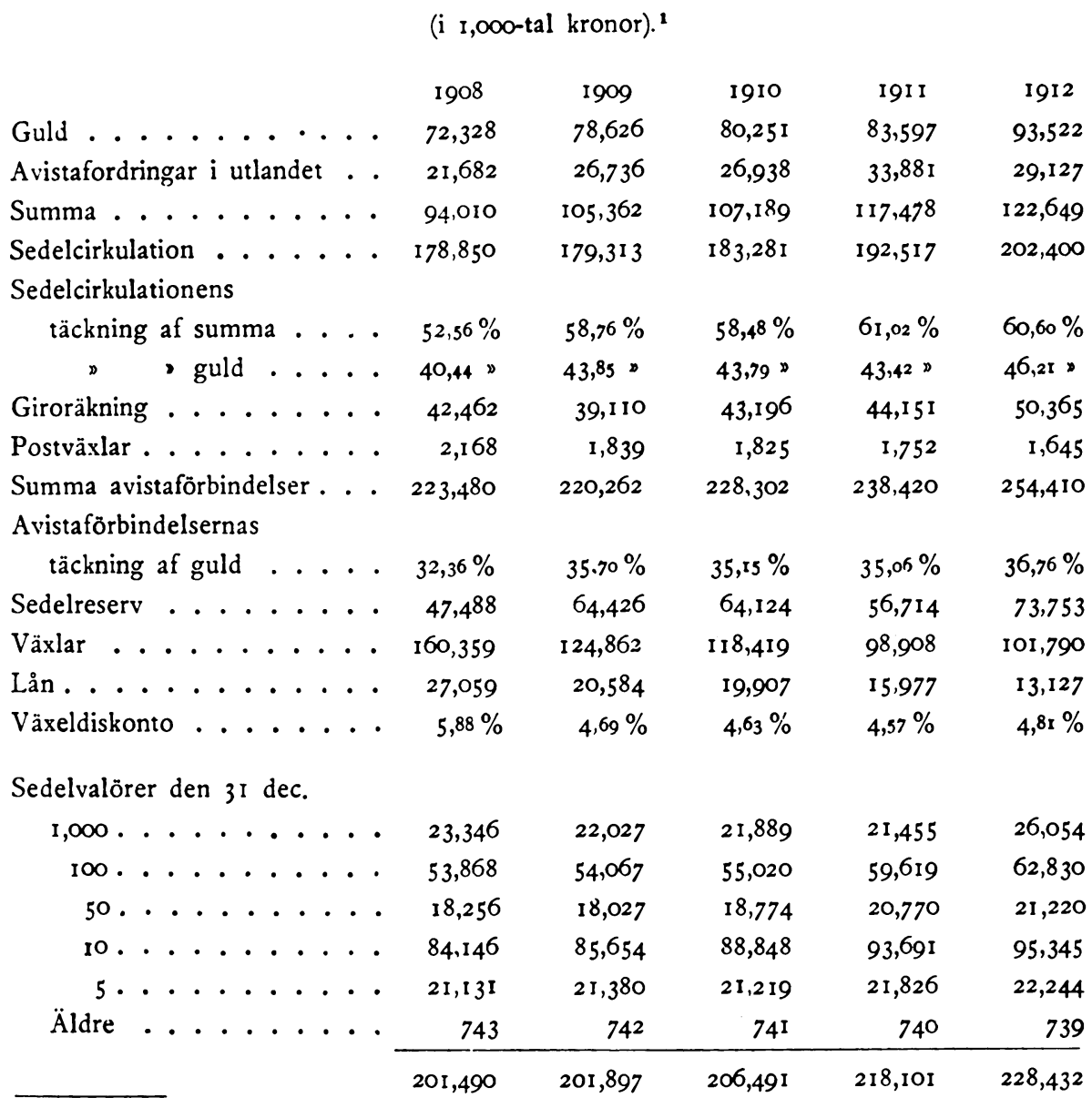

${ }^{1}$ Enligt bankens årsböcker. 


\section{Norges Bank}

(i I,ooo-tal kronor).

\begin{tabular}{|c|c|c|c|c|c|}
\hline & 1908 & 1909 & IOIO & I9II & 1912 \\
\hline Guld $^{\prime} \ldots \ldots$ & 27,453 & 29,534 & 31,705 & 36,383 & 37,365 \\
\hline \multicolumn{6}{|l|}{ Behållning i Sverige och Dan- } \\
\hline $\operatorname{mark}^{1} \ldots \ldots \ldots$ & 1,932 & $\mathrm{I}, 083$ & 2,043 & $\mathrm{I}, 729$ & I,339 \\
\hline Öfrig behållning i utlandet ${ }^{1} \cdot$. & I6,868 & I 7,227 & $\mathrm{I} 7, \mathrm{I} 49$ & 19,450 & I9,624 \\
\hline Summa guldbehållning. . . • & 46,253 & 47,844 & 50,897 & 57,562 & 58,328 \\
\hline Sedelcirkulation $^{1} \ldots$ & 73,355 & 75,374 & $8 \mathrm{I}, 224$ & 88,323 & 94,606 \\
\hline \multicolumn{6}{|l|}{ Sedelcirkulationens } \\
\hline täckning af behållning . . . & $63,05 \%$ & $63,4^{8} \%$ & $62,66 \%$ & $65,17 \%$ & $61,65 \%$ \\
\hline$" \quad$ guld ..... & $37,42 》$ & $39, \mathbf{1 8} »$ & $39,03 »$ & $4 \mathrm{I}, \mathrm{r} 9 》$ & $39,50 "$ \\
\hline Sedelreserv $^{1} \ldots \ldots$ & 7,899 & 7,470 & 4,673 & 4,239 & $2,60 \mathrm{I}$ \\
\hline Öfriga avistaförbindelser ${ }^{21} .$. & $7,56 \mathrm{I}$ & 7,145 & 7,244 & $7,77 \mathrm{I}$ & 8,038 \\
\hline Summa avistaförbindelser ... & 80,916 & 82,5 I9 & 88,468 & 96,094 & I02,644 \\
\hline \multicolumn{6}{|l|}{ Avistaförbindelsernas täckning af } \\
\hline . . . • • & $33,93 \%$ & $35,79 \%$ & $35,84 \%$ & $37,86 \%$ & $36,40 \%$ \\
\hline 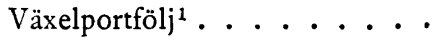 & 33,100 & $37, \mathrm{I} 44$ & $40,93 \mathrm{I}$ & 44,270 & 53,077 \\
\hline Växeldiskonto $^{1} \ldots \ldots$ & $5,344 \%$ & $4,543 \%$ & $4,500 \%$ & $4,628 \%$ & $5,383 \%$ \\
\hline
\end{tabular}

Sedelvalörer ${ }^{3}$ den 3 I december

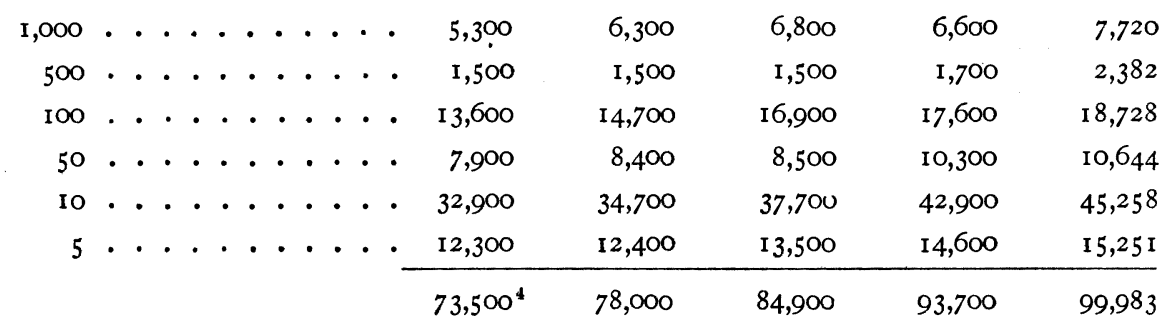

1 Uträkningar enligt månadsrapporterna.

2 Folio.

${ }^{3}$ Enligt bankens årsberättelser.

4 Afgår kassa 1908: 687; 1909: 506; 1910: 618; 1911: 827; 1912: 707. 


\section{Nationalbanken i Köpenhamn}

(i I, 000-tal kronor).

Medeltal pr kalenderår.

\begin{tabular}{|c|c|c|c|c|c|}
\hline & 1908 & 1909 & I910 & I9I I & 1912 \\
\hline${\text { Guld }{ }^{1} \ldots \ldots}^{\ldots}$. . . . & - & 69,189 & 66,600 & 67,682 & 68,296 \\
\hline Skiljemynt $^{1}$. . . . . & - & $6,33 \mathrm{I}$ & 6,3 I 2 & 6,2 I 5 & 6,193 \\
\hline Summa metall . . . . . & 72,857 & 75,520 & 72,912 & 73,897 & 74,489 \\
\hline Fordran af $(+)$ eller skuld till & & & & & \\
\hline$(-)$ utlandet $^{1} \ldots \ldots$ & -397 & $-1,822$ & -546 & $-\mathrm{I}, 646$ & $+98 \mathrm{I}$ \\
\hline Summa metallfond $^{2} \cdot \ldots \cdot$ & 72,460 & 73,698 & 72,366 & 72,251 & 75,470 \\
\hline Sedelcirkulation $^{2}$. . . . & I 21,438 & I 22,095 & I 27,799 & I 32,765 & I 39,037 \\
\hline Sedelcirkulationens & & & & & \\
\hline täckning af metallfond. . . & $59,67 \%$ & $60,36 \%$ & $56,62 \%$ & $54,42 \%$ & $54,28 \%$ \\
\hline, metall .... & $60, \infty 》$ & $61,85>$ & $57,05 »$ & $55,66 »$ & $53,57 》$ \\
\hline$» \quad$ guld. . . . . & 一 & $56,67 \gg$ & $52, \mathrm{Ir} \gg$ & 50,98 & $49,12 "$ \\
\hline Sedelreserv $^{2}$. . . . . & $3,5^{14}$ & 25,301 & I6,933 & I I,737 & I I,903 \\
\hline Kontokuranter $^{2}$. . . . & 4,127 & 4,844 & 5,969 & $5,56 \mathrm{I}$ & 5,279 \\
\hline Summa avistaförbindelser . . • & 125,565 & I26,939 & I 33,768 & I 38,326 & 144,316 \\
\hline Avistaförbindelsernas & & & & & \\
\hline täckning af metallfond .. & $57,7 \mathrm{r} \%$ & $58,06 \%$ & $54,10 \%$ & $52,23 \%$ & $52,29 \%$ \\
\hline " metall. . . . & $58,02 ”$ & $59,49 \gg$ & $54,5 \times ”$ & $53,42 \bowtie$ & $51,62 »$ \\
\hline , guld.. & 一 & $54,51 »$ & $49,79 ”$ & 48,93 » & 47,32 \\
\hline Jtländska växlar, utländska kor- & & & & & \\
\hline respondenter ${ }^{2} \cdot \ldots \cdot \cdot$ & I 3,755 & 14,901 & 16,963 & 25,569 & 26,023 \\
\hline Växlar $^{2} \ldots \ldots \cdot \ldots$ & 35,5 I 8 & 30,328 & 27,677 & 26,555 & 29,067 \\
\hline$\cdots \cdots \cdots$ & $3 \mathrm{I}, 3 \mathrm{O} 3$ & 34,970 & $47,6 \mathrm{ro}$ & 44,878 & 41,872 \\
\hline Växeldiskonto & $6,1,36 \%$ & $4,942 \%$ & $5,000 \%$ & $4,624 \%$ & $5,062 \%$ \\
\hline
\end{tabular}

Sedelvalörer ${ }^{8}$ den 3 I juli

\begin{tabular}{|c|c|c|c|c|c|c|}
\hline 500 & $\cdots \cdots \cdots$ & 12,644 & I I, 769 & IO,75.4 & I4,4 I9 & I 5,470 \\
\hline 100 & 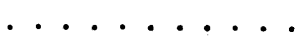 & 43,158 & 44,740 & 45,350 & 48,8 I 5 & 50,230 \\
\hline 50 & - & 10,986 & I0,445 & II, 175 & 10,855 & 12,322 \\
\hline IO & . . & 59,172 & 59,731 & 61,876 & 65,686 & 67,965 \\
\hline \multirow[t]{2}{*}{5} & $\cdot \cdot \cdot \cdot$ & 7,040 & $7 ; 315$ & 7,845 & 8,225 & 9,013 \\
\hline & & $133,000^{4}$ & I 34,000 & I 37,000 & $\mathbf{1 4 8 , 0 0 0}$ & I 55,000 \\
\hline
\end{tabular}

1 Uträkningar enligt af banken enskildt meddelade uppgifter.

2 " månadsrapporterna.

${ }^{8}$ Enligt bankens årsberättelser.

4 Afgår inneliggande i kassa 1908: 10,884; 1909: I1,562; 1910: 11,394; 1911: 12,010; I912: 14,740. 


\title{
De Nederlandsche Bank
}

(i I, , oo-tal Fl.).

\author{
Medeltal pr kalenderår.
}

\begin{tabular}{|c|c|c|c|c|c|}
\hline & I908 & 1909 & I9IO & I9II & I912 \\
\hline Guld $^{1} \cdot \ldots$ & 93,378 & I I 9,569 & I I 4,668 & I 36,869 & I 48,815 \\
\hline Silfver $^{1}$. . . . . . . & 50,396 & 42,226 & 27,963 & 21,571 & 10,470 \\
\hline Summa metall . . . . . & 143,774 & I6I ,795 & $\mathrm{I} 42,63 \mathrm{I}$ & I 58,440 & 159,285 \\
\hline Sedelcirkulation $^{1} \ldots \ldots$ & 268,767 & 280,448 & $278,6 \mathrm{I} 6$ & 290,488 & 301,610 \\
\hline \multicolumn{6}{|l|}{ Sedelcirkulationens } \\
\hline täckning af metall. . . . & $53,49 \%$ & $57,69 \%$ & $5 I, 1_{9} \%$ & $54,54 \%$ & $52,8 \mathrm{I} \%$ \\
\hline , guld. . . . . . & $34,74 »$ & $42,63 》$ & $4 \mathrm{I}, \mathrm{16} 》$ & $47,12 》$ & $49,34 »$ \\
\hline Avistaförbindelser till staten ${ }^{1}$. & - & - & - & I,989 & 2,342 \\
\hline Öfriga avistaförbindelser ${ }^{1} \cdot \cdots$ & 6,528 & 7,360 & 6,438 & $6,69 \mathrm{I}$ & 5,532 \\
\hline Summa avistaförbindelser. . . & 275,295 & 287,808 & 285,054 & $299, \mathrm{I} 68$ & 309,484 \\
\hline \multicolumn{6}{|l|}{ Avistaförbindelsernas } \\
\hline täckning af metall. & $52,23 \%$ & $56,22 \%$ & $50,04 \%$ & $52,96 \%$ & $5 \mathrm{I}, 47 \%$ \\
\hline$"$ " guld. . . . . & 33,92 & $4 \mathrm{I}, 54 »$ & $40,23 \gg$ & $45,75 \gg$ & $48,08 »$ \\
\hline Disponibelt metallsaldo ${ }^{1} \ldots$ & 33,556 & 46,526 & 28,5 I I & 38,624 & 35,301 \\
\hline Utländska växlar ${ }^{1}$. . . & I I, 873 & 19,624 & I I, I 26 & I 5,493 & $\mathrm{I} 8,270$ \\
\hline Växlar $^{1} \ldots \ldots \ldots$ & $56,94 \mathrm{I}$ & 48,693 & 59,277 & 59,399 & 70,736 \\
\hline Lån och löpande räkning ${ }^{1}$. . & 69,599 & $6 \mathrm{I}, 240$ & 76,210 & 76,092 & 75,454 \\
\hline${\text { Växeldiskonto }{ }^{2} \ldots} \ldots \ldots$ & $3,38 \%$ & $2,88 \%$ & $4,23 \%$ & $3,45 \%$ & $4,00 \%$ \\
\hline
\end{tabular}

Sedelvalörer ${ }^{2}$ den 31 mars

\begin{tabular}{|c|c|c|c|c|c|}
\hline I,000 . . . . . . & 43,267 & 46,9 I 2 & $47,95 \mathrm{I}$ & 45,089 & 44,518 \\
\hline $500 \ldots . \ldots$ & 6 & 6 & 6 & 6 & 6 \\
\hline $300 . \cdot \cdots \cdot \cdot \cdots \cdot$ & 20,407 & 21,127 & 20,826 & 20,620 & 22,654 \\
\hline 200 . . . . . . . & 19,094 & I9,6I 6 & I9, I I 5 & I 8,696 & 20,549 \\
\hline 100..... & 59,152 & $60,75^{2}$ & 62,189 & 63,006 & $67,77 \mathrm{I}$ \\
\hline $80 \cdot \cdot \cdot \cdot \cdot \cdot \cdot \cdot$ & I & I & I & I & I \\
\hline $60 . . . \cdot$ & $34, \mathrm{I} 4 \mathrm{O}$ & 34,009 & $34, \mathrm{I} 79$ & 34,6 I 4 & 35,795 \\
\hline $40 \ldots . . .$. & 26,274 & $26,05 \mathrm{I}$ & 26,259 & 26,719 & 27,531 \\
\hline $25 \ldots \ldots$ & 39,168 & 40,015 & $4 \mathrm{I}, 43 \mathrm{I}$ & $42,73 \mathrm{I}$ & 44,797 \\
\hline \multirow[t]{2}{*}{ го. . . . . . } & 24,439 & 25,089 & 28,596 & $3 \mathrm{I}, 788$ & 35,343 \\
\hline & 265,948 & 273,578 & 280,553 & 283,270 & 298,965 \\
\hline
\end{tabular}

1 Uträkningar enligt veckorapporterna.

2 Enligt bankens årsberättelser. 


\section{Banque Nationale de Belgique}

(i I,ooo-tal frcs).

\begin{tabular}{|c|c|c|c|c|c|}
\hline & I 908 & I909 & I9IO & I9I I & 1912 \\
\hline Guld $^{1} \cdot \ldots \cdot \ldots$ & - & - & - & I 75,66I & I 98,200 \\
\hline Silfver $^{1} \cdot \ldots \cdot \ldots$ & - & - & - & $67,58 \mathrm{I}$ & 67,011 \\
\hline Summa metall ${ }^{1} \ldots \ldots$ & I 5 I,947 & I 58,958 & I 79,660 & 243,242 & $265,2 \mathrm{II}$ \\
\hline Utländska valutor ${ }^{1} \ldots \ldots$ & $\mathrm{I} 8 \mathrm{I}, 56 \mathrm{I}$ & I 87,086 & I 55,859 & 1 51,696 & 146,856 \\
\hline Summa kassa . . . . . & 333,508 & 346,044 & 335,5 I9 & 394,938 & 412,067 \\
\hline Sedelcirkulation $^{2}$. . . . & 753,080 & 770,402 & 826,272 & 882,190 & 946, I 50 \\
\hline \multicolumn{6}{|l|}{ Sedelcirkulationens } \\
\hline täckning af kassa . . . & $44,29 \%$ & $44,92 \%$ & $40,6 \mathrm{x} \%$ & $44,77 \%$ & $43,55 \%$ \\
\hline$"$ metall ... & $20,18 》$ & $20,63 »$ & $21,74 »$ & $27,57 》$ & $28,03 》$ \\
\hline$»$ guld $\ldots$. & - & - & - & I9,9I 》 & 20,95 * \\
\hline Avistaförbindelser till staten ${ }^{2}$ & I 8,985 & 17,562 & I 5,934 & 24,695 & 20,449 \\
\hline Öfriga avistaförbindelser ${ }^{2} \cdot$. & $63,26_{3}$ & 66,976 & 65,696 & 82,020 & 77,951 \\
\hline Summa avistaförbindelser $\cdot$ & 835,328 & 854,940 & 907,902 & 988,905 & $\mathrm{I}, 044,55^{\circ}$ \\
\hline \multicolumn{6}{|l|}{ Avistaförbindelsernas } \\
\hline täckning af metall & I8, rg \% & I $8,59 \%$ & $19,79 \%$ & $24,60 \%$ & $25,39 \%$ \\
\hline$\bowtie$ guld .... & - & - & - & $17,76 "$ & I $8,97 "$ \\
\hline$\cdots \cdot \cdot \cdot$ & 601,832 & 612,629 & $63 I, 854$ & 479,744 & 512,579 \\
\hline$\cdot \cdot \cdot \cdot$ & 55,758 & 53,018 & 67,656 & 84,280 & 85,995 \\
\hline Växeldiskonto $^{2} \cdot \cdot \cdot \cdot \cdot \cdot$ & $3,56 \%$ & $3, \mathrm{rr} \%$ & $4,12 \%$ & $4,15 \%$ & $4,49 \%$ \\
\hline
\end{tabular}

Sedelvalörer, ${ }^{2}$ i medeltal.

\begin{tabular}{|c|c|c|c|c|c|}
\hline $\mathrm{I}, 000 \cdots \cdot \cdots \cdot \cdots$ & 160,267 & I 82,275 & 198,339 & 209,932 & 216,173 \\
\hline $500 \cdot \cdot \cdot \cdot \cdot \cdot \cdot$ & 72,146 & 54,928 & 51,400 & 53,106 & 52,702 \\
\hline $100 \cdot \cdots \cdot \cdots$ & 297,698 & 301,656 & 320,958 & $342,26_{3}$ & 370,955 \\
\hline 50. & 73,609 & 76,520 & 83,983 & 90,519 & 100,432 \\
\hline \multirow[t]{2}{*}{$20 \ldots \ldots \cdot$} & I 49,360 & 155,023 & I 71,592 & 186,370 & 205,888 \\
\hline & 753,080 & 770,402 & 826,272 & 882, I 90 & 946, I 50 \\
\hline
\end{tabular}

1 Uträkningar enligt veckorapporterna.

${ }^{2}$ Enligt bankens årsberättelser. 


\section{Schweizerische Nationalbank}

\begin{tabular}{|c|c|c|c|c|c|}
\hline & I908 & I909 & I9IO & I9II & 1912 \\
\hline Guld $^{1}$. . . . . . . & 98,329 & I 21,492 & I 43,346 & I $58,52 \mathrm{I}$ & I 68,084 \\
\hline Silfver $(5 \text {-frcs-stycken })^{1} \ldots$. & 8,090 & 15,209 & I6,o76 & $\mathrm{I} 4,790$ & I 3,049 \\
\hline Summa metall . . . . . & 106,419 & I $36,70 \mathrm{I}$ & I 59,422 & I 73,3 I I & I $8 \mathrm{I}, \mathrm{I} 33$ \\
\hline Sedelcirkulation $^{1}$. . . . . & 145,870 & I9I, 428 & $24 \mathrm{I}, 422$ & 253,573 & 269,340 \\
\hline \multicolumn{6}{|l|}{ Sedelcirkulationens } \\
\hline täckning af metall ${ }^{1} \ldots$. & $72,95 \%$ & $71,41 \%$ & $66,03 \%$ & $68,35 \%$ & $67,25 \%$ \\
\hline$» \quad »$ guld ..... & $67,41 »$ & $63,47 »$ & $59,38 》$ & $62,5 \mathrm{x} \gg$ & $62,41 》$ \\
\hline Öfriga avistaförbindelser ${ }^{12}$. & $25,4 \mathrm{I} 4$ & 36,993 & 59,896 & 58,690 & 43, I I 8 \\
\hline Summa avistaförbindelser . . . & I 7 I, 284 & $228,42 \mathrm{I}$ & 301,318 & 312,263 & $312,45^{8}$ \\
\hline \multicolumn{6}{|l|}{ Avistaförbindelsernas } \\
\hline täckning af metall . . . . & $62,13 \%$ & $59,85 \%$ & $5 z, 9 \mathrm{I} \%$ & $55,50 \%$ & $57,97 \%$ \\
\hline$\gg \quad »$ guld . . . . & $57,4 \mathrm{I} \gg$ & $53,19 》$ & $47,57 »$ & $50,77 刃$ & $53,79 \gg$ \\
\hline Växlar $^{1} \ldots$. . . . . & 63,007 & 85,037 & I09,493 & I I I, 335 & I I I, 589 \\
\hline $\operatorname{Lån}^{1} \cdot \ldots$. . . . . & 2,529 & 4,410 & 5,933 & 7,037 & $\mathrm{I} 2,232$ \\
\hline Växeldiskonto $^{1} \cdot \ldots \cdot \cdots$ & $3,73 \%$ & $3,22 \%$ & $3,5 \mathrm{x} \%$ & $3,70 \%$ & $4,20 \%$ \\
\hline \multicolumn{6}{|l|}{ Sedelvalörer ${ }^{1}$ den 3 I december. } \\
\hline 1,000 . . . . . . & I $3, \mathrm{I} 53$ & I 8,644 & I $8,8 \mathrm{I} 6$ & 22,255 & 23,824 \\
\hline $500 \ldots . \cdots$ & I 3,640 & 22,326 & 26,035 & 25,914 & $26,85^{6}$ \\
\hline 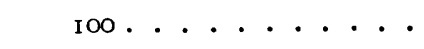 & 107,948 & I 38,949 & I 57,353 & I65,532 & I 77,076 \\
\hline \multirow[t]{2}{*}{$50 \cdots \cdot \cdots \cdot$} & 69,3 I 5 & 81,597 & 95,008 & IOI, 055 & I I I 484 \\
\hline & 204,056 & $26 I, 516$ & 297,212 & $314,75^{6}$ & 339,240 \\
\hline
\end{tabular}

${ }^{1}$ Enligt bankens årsberättelser.

${ }^{2}$ Giro och deponenter. 


\section{Banque Nationale privilégiée du Royaume de Serbie}

(i 1,000 -tal dinarer).

\begin{tabular}{|c|c|c|c|c|c|}
\hline & 1908 & 1909 & I910 & I9I I & $1912^{2}$ \\
\hline Guld $^{1} \ldots \ldots$ & 12,250 & 14,284 & $2 \mathrm{I}, 72 \mathrm{I}$ & 28,185 & 42,629 \\
\hline Silfver $^{1} \ldots \ldots \ldots$ & 7,389 & $6,89 \mathrm{I}$ & $6,88 \mathbf{I}$ & 6,960 & 5,986 \\
\hline Summa metall . . . . . . & 19,639 & 21,175 & 28,602 & 35,145 & 48,6 I 5 \\
\hline Sedelcirkulation: guld ${ }^{1} \ldots$ & 4,335 & $3,36 \mathbf{I}$ & 5,155 & I0,593 & 9,963 \\
\hline silfver $^{1} .$. & 35,439 & 46,985 & $45,75^{8}$ & 49,358 & 62,340 \\
\hline $\begin{array}{l}\text { Summa sedelcirkulation } \cdots \\
\text { Sedelcirkulationens }\end{array}$ & 39,774 & 50,346 & 50,913 & $59,95 \mathbf{I}$ & 72 \\
\hline täckning af metall . . . & $49,38 \%$ & $42,06 \%$ & $56,18 \%$ & $58,62 \%$ & $67,24 \%$ \\
\hline$» \quad »$ guld $\ldots .$. & $30,80 》$ & $28,37 》$ & $42,66 »$ & 47, or $»$ & $58,96 »$ \\
\hline Öfriga avistaförbindelser ${ }^{18} \cdot \cdot$ & $\mathrm{I}, 483$ & 4, OI 5 & 3,288 & 3,369 & 5,390 \\
\hline $\begin{array}{l}\text { Summa avistaförbindelser } \cdot \cdot \cdot \\
\text { Avistaförbindelsernas }\end{array}$ & 41,257 & $54,36 \mathrm{I}$ & 54,201 & 63,320 & 77,693 \\
\hline täckning af metall. . . . & $47,60 \%$ & $38,95 \%$ & $52,77 \%$ & $55,50 \%$ & $62,57 \%$ \\
\hline$\triangleright \quad "$ guld $\ldots$. & $29,69 》$ & $26,28 》$ & $40,07 \gg$ & $44,50 »$ & $54,87 \gg$ \\
\hline $\operatorname{Växlar}^{1} \cdot \cdot \cdot \cdot \cdot \cdot \cdot \cdot$ & 8,073 & 8,785 & 7,659 & 7,698 & 6,195 \\
\hline $\operatorname{Lån}^{1} \ldots \ldots \ldots$ & 6,065 & 6,908 & 4,252 & 2,083 & 2,502 \\
\hline Utländska korrespondenter ${ }^{1}$. . & 4,334 & 7,777 & 7,302 & 7,257 & I I 672 \\
\hline Löpande räkning ${ }^{1} \ldots \ldots$ & 9,2 I 8 & 9,724 & 9,878 & 16,985 & 23,507 \\
\hline
\end{tabular}

Sedelvalörer ${ }^{1}$ den 3 I december

Guld 100 d. $\cdot \cdots \cdot \cdot \cdot \cdot$

\begin{tabular}{rrrrrrr} 
roo d. . . . . . & 600 & 230 & 69 & 32 & - \\
5o d. . . . . . & I & I & I & - & - \\
20 d. . . . . & 2,773 & 3,233 & 6,967 & 13,949 & - \\
\hline Summa & 3,374 & 3,464 & 7,037 & 13,981 & -
\end{tabular}

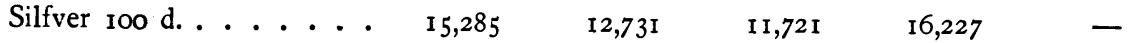

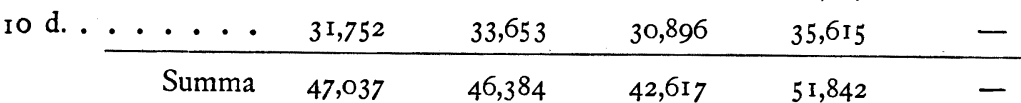

${ }^{1}$ Enligt bankens årsberättelser.

2 Uträkningar enligt veckorapporterna.

${ }^{3}$ Comptes courants (passif). 


\section{Banque Nationale de Bulgarie}

(i I,ooo-tal levs).

\begin{tabular}{|c|c|c|c|c|c|}
\hline & 1908 & 1909 & I9IO & I9I I & $1912^{3}$ \\
\hline$\ldots \ldots \ldots$ & 23,198 & 25,322 & 31,913 & $35, \mathrm{I} 32$ & 44,289 \\
\hline . . . . . & 16,233 & 16,072 & I $8,89 \mathrm{I}$ & 20,774 & I 8,450 \\
\hline Summa metall . & 39,43 I & $4 \mathrm{I}, 394$ & 50,804 & 55,906 & 62,739 \\
\hline Sedelcirkulation: guld ${ }^{1}$ & 44,869 & 55,967 & 53,362 & 74,234 & - \\
\hline silfver ${ }^{1}$. . & I 5,6 I I & 21,957 & 20,678 & 24 , II 2 & - \\
\hline Summa sedelcirkulation & 60,480 & 77,924 & 74,040 & 98,346 & I 30,603 \\
\hline Sedelcirkulationens täckning af metall & $65,20 \%$ & $53, \mathrm{r} 2 \%$ & $68,62 \%$ & $56,85 \%$ & $48,04 \%$ \\
\hline , , guld & 38,36 & $32,50 »$ & $43,10 》$ & $35,72 》$ & $33,9 \pm »$ \\
\hline Öfriga avistaförbindelser ${ }^{12} \ldots \ldots$ & 一 & 23,859 & 16,084 & I I,798 & - \\
\hline Summa avistaförbindelser . & - & IOI,783 & 90,124 & I I O, I 44 & - \\
\hline Avistaförbindelsernas täckn. af metall & 一 & $40,67 \%$ & $56,37 \%$ & $50,76 \%$ & - \\
\hline " guld . & 一 & $24,88 》$ & $35,4 \mathrm{I} \gg$ & $3 \mathrm{I}, 90$ * & - \\
\hline Växlar ${ }^{1}$ & 23,854 & 28,5 I I & 38,424 & 43,173 & - \\
\hline Lån och löpande räkning ${ }^{13}$. & 38,463 & 37,156 & 49,173 & 55,132 & - \\
\hline
\end{tabular}

\section{Banque Nationale de Roumanie}

(i I,ooo-tal lei).

\begin{tabular}{|c|c|c|c|c|c|}
\hline & 1908 & 1909 & I9IO & I9I I & 1912 \\
\hline $1 \mathrm{ld}^{4}$ & $9 \mathrm{I}, 4 \mathrm{I} 2$ & $9 \mathrm{I}, 2 \mathrm{I} 8$ & 102,355 & I 35,485 & I 57, I 53 \\
\hline & $\mathrm{I}, \mathrm{I} 5 \mathrm{O}$ & 852 & $\mathrm{I}, 005$ & 850 & 709 \\
\hline metall & 92,562 & 92,070 & 103,360 & I 36,33 & 57,862 \\
\hline lgodohafvanden $\mathrm{i}$ utlandet & $37,67 \mathrm{I}^{1}$ & $36,488^{4}$ & $42,395^{4}$ & $54,474^{4}$ & $63, c$ \\
\hline a kassa . . . . . . & I 30,233 & I 28,558 & I 45,755 & 190,809 & 220,954 \\
\hline delcirkulation för staten ${ }^{1}$ & I4,694 & 14,468 & 14,2 IO & $\mathrm{I} 3,750$ & 13,325 \\
\hline "rörelsen ${ }^{1}$ & $248,36 \mathrm{I}$ & 257, i 36 & 285,239 & 368,6 Io & 449,92 \\
\hline mma sedelcirkulation .... & 263,055 & 271,604 & 299,449 & 382,360 & 463,246 \\
\hline cirkulationens täckn. af kassa & $49,5 \mathrm{I} \%$ & $47,33 \%$ & $48,67 \%$ & $49,90 \%$ & 47,70 \\
\hline$» \quad$ metall & $35,19 »$ & $33,90 》$ & $34,52 \%$ & $35,66 »$ & 34,08 \\
\hline $\begin{array}{l}" » \text { guld } \\
. . .\end{array}$ & $\begin{array}{l}34,75 » \\
69,500\end{array}$ & $\begin{array}{r}33,58 》 \\
66,506\end{array}$ & $\begin{array}{c}34, \mathrm{r} 8 》 \\
88, \mathrm{OI} 3\end{array}$ & $\begin{array}{l}35,43 » \\
\text { I } 32,888\end{array}$ & $\begin{array}{l}33,92 \\
184,57\end{array}$ \\
\hline eldiskonto & $\begin{array}{l}27,932 \\
5,18 \%\end{array}$ & $\begin{array}{l}31,327 \\
5,00 \%\end{array}$ & $\begin{array}{l}28,939 \\
5,00 \%\end{array}$ & $\begin{array}{l}49,512 \\
5,00 \%\end{array}$ & $\begin{array}{l}80,5 \\
5,20\end{array}$ \\
\hline
\end{tabular}

Sedelvalörer ${ }^{1}$ den 3 I december

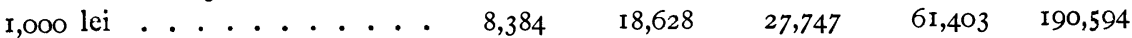

$$
\begin{aligned}
& \text { I00 } " \ldots \ldots \ldots 224,236 \quad 239,035 \quad 286,506 \quad 345,88 \mathrm{I} \quad 332,328 \\
& 20 \text { 20......... } \begin{array}{rrrrr}
66,667 & 67,193 & 84,335 & 102,298 & 123,024 \\
\hline
\end{array}
\end{aligned}
$$

$299,297 \quad 324,856 \quad 39,8,588 \quad 509,582 \quad 645,946$

1 Enligt bankens årsberättelser.

2 Comptes courants sans intérets.

${ }^{3}$ Comptes courants spéciaux sous garantie d'effets déposés, Comptes courants spéciaux sous garantie de deux personnes, Comptes et avances sur dépôt de titre, Comptes et avances sur dépôt de marchandises et de connaissements.

4 Uträkningar enligt veckorapporterna $\mathrm{i}$ »Die Bank». 


\section{Banco de España}

(i I,000 tal pesetas).

\begin{tabular}{|c|c|c|c|c|}
\hline & 1909 & I9Io & I9I I & 1912 \\
\hline Guld $^{1} \ldots \ldots$ & 399,268 & 406,825 & 414,436 & 426,629 \\
\hline Silfver $^{1} \ldots \ldots \ldots$ & 797,155 & 773,247 & 770,292 & 749,737 \\
\hline . . . . . & $1,196,423$ & $1,180,072$ & $1,184,728$ & $\mathrm{I}, \mathrm{I} 76,366$ \\
\hline Sedelcirkulation $^{1} \ldots \ldots$ & $\mathrm{I}, 67 \mathrm{I}, 8 \mathrm{II}$ & $\mathrm{I}, 704,080$ & $1,735,465$ & $\mathrm{I}, 8 \mathrm{r} 6,8 \mathrm{I} 9$ \\
\hline \multicolumn{5}{|l|}{ Sedelcirkulationens } \\
\hline täckning af metall & $71,56 \%$ & $69,25 \%$ & $68,27 \%$ & $64,75 \%$ \\
\hline$" \quad$ "guld $\ldots \ldots \ldots$ & $23,88 "$ & $23,87 »$ & $23,88 "$ & $23,48 \gg$ \\
\hline Fordringar i utlandet ${ }^{1} \ldots \ldots$ & 95,669 & 1 34,312 & I36,4 I9 & 165,813 \\
\hline $\begin{array}{l}\text { Växlar }^{1} \ldots \cdot \cdots \cdots \\
\text { Lån och löpande räkning }{ }^{1}\end{array}$ & $29 \mathrm{I}, 76 \mathrm{I}$ & 286,306 & 283,299 & 271,373 \\
\hline Lån och löpande räkning ${ }^{1} \ldots$. . & $47 \mathrm{I}, 9 \mathrm{II}$ & 479,864 & 496,069 & 402,405 \\
\hline 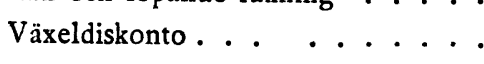 & $4,500 \%$ & $4,500 \%$ & $4,500 \%$ & $4,500 \%$ \\
\hline \multicolumn{5}{|l|}{ Sedelvalörer $^{2}$ den 31 december. } \\
\hline $\mathrm{I}, 000 \ldots \ldots$ & $44 \mathrm{I}, 957$ & 442,095 & 449,027 & $465, \mathrm{I} 4 \mathrm{I}$ \\
\hline $500 \cdot \cdots \cdot \cdot \cdot \cdot \cdot$ & 196,009 & 210,780 & 214,434 & 230,849 \\
\hline $250 \cdot \cdots \cdot \cdot \cdot \cdot \cdot \cdot$ & 85 & 85 & 84 & 84 \\
\hline $125 \cdot \cdots \cdot \cdots \cdot \cdots$ & I 27 & I 27 & I27 & 127 \\
\hline $100 \cdot \cdot \cdot \cdot \cdot \cdots \cdot$ & 623,384 & 656,557 & 693,608 & 740,199 \\
\hline $50 \cdot \cdot \cdot \cdot \cdot \cdot \cdot \cdot \cdot$ & 301,767 & 298,748 & 297,963 & 316,165 \\
\hline $25 \cdot \cdots \cdot \cdots \cdot \cdots \cdot$ & 107,669 & 106,833 & 107,529 & I 10,258 \\
\hline & I,670,998 & 15,225 & $\mathrm{I}, 762,772$ & $I, 862,823$ \\
\hline
\end{tabular}

\section{Banco de Portugal}

(i millioner Réis).

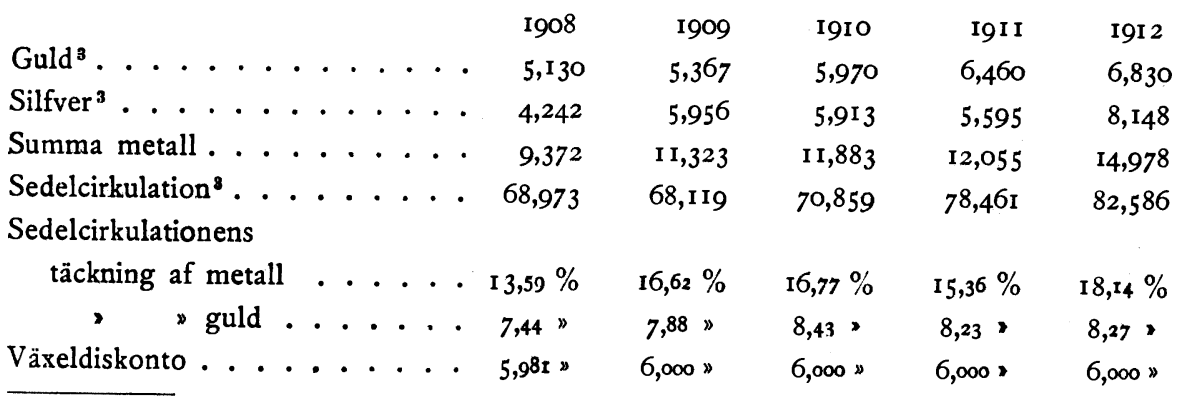

1 Uträkningar enligt veckorapporterna.

${ }^{2}$ Enligt bankens årsberättelser.

"Uträkningar enligt veckorapporterna i "Die Bank". 


\section{Sedelcirkulationens täckning af täckningsvalutor (enligt lagens föreskrift eller vedertagen praxis) i procent.}

\begin{tabular}{|c|c|c|c|c|c|}
\hline & 1908 & 1909 & I910 & I9I I & I9I2 \\
\hline Bank of England . . . . . . & I29,II & 128,07 & I 30,75 & I 33,39 & I 34,55 \\
\hline Banque de France... & 81,52 & 89,06 & $8 \mathrm{I}, 99$ & 76,98 & 75,67 \\
\hline Reichsbank . . & 71,26 & 70,64 & 69,75 & 71,23 & 69,74 \\
\hline Österreichisch-Ungarische Bank . & 80,75 & 85,25 & $8 \mathrm{I}, 9 \mathrm{I}$ & 75,36 & 69,62 \\
\hline 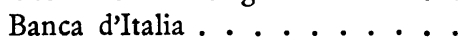 & $8 \mathrm{I}, 75$ & 82,97 & 79,78 & 77,58 & $75,2 \mathrm{I}$ \\
\hline Ryska Riksbanken . . . . . . . & I I 3,35 & I 21,97 & 127,57 & I 20,58 & II 5,73 \\
\hline Finlands Bank . . . . . . . & II 6,30 & I 22,76 & I I I, 66 & I 22,82 & I I 3,39 \\
\hline Sveriges Riksbank . . . . . & 52,56 & 58,76 & 58,48 & 61,02 & 60,60 \\
\hline Norges Bank ... & 63,05 & 63,48 & 62,66 & 65,17 & $6 \mathrm{r}, 65$ \\
\hline Nationalbanken i Köpenhamn . & 59,67 & 60,36 & 56,62 & 54,42 & 54,28 \\
\hline De Nederlandsche Bank . . . & 53,49 & 57,69 & $5^{1,19}$ & 54,54 & $52,8 \mathrm{I}$ \\
\hline Banque Nationale de Belgique . . & 44,29 & 44,92 & $40,6 \mathbf{z}$ & 44,77 & 43,55 \\
\hline Schweizerische Nationalbank . . & 72,95 & $7 \mathrm{I}, \mathbf{4} \mathrm{I}$ & 66,03 & 68,35 & 67,25 \\
\hline Banque Nationale privilégiée du & & & & & \\
\hline Royaume de Serbie . . . . & 49,38 & 42,06 & 56,18 & 58,62 & 67,24 \\
\hline Banque Nationale de Bulgarie . & 65,20 & 53,12 & 68,62 & 56,85 & 48,04 \\
\hline Banque Nationale de Roumanie. & $49,5 \mathrm{I}$ & 47,33 & 48,67 & 49,90 & 47,70 \\
\hline Banco de España . . . . . & - & 71,56 & 69,25 & 68,27 & 64,75 \\
\hline Banco de Portugal. . & 13,59 & 16,62 & 16,77 & I 5,36 & 18,14 \\
\hline
\end{tabular}

\section{Sedelcirkulationens täckning af metall i procent.}

\begin{tabular}{|c|c|c|c|c|c|}
\hline & I908 & 1909 & I9IO & I9I I & I9I 2 \\
\hline Bank of England & I29,II & 128,07 & I 30,75 & 133,39 & I 34,55 \\
\hline Banque de France . . . . . & 81,52 & 89,06 & 81,99 & 76,98 & 75,67 \\
\hline Reichsbank . . . . . . & 66,86 & 66,37 & 65,74 & 67,88 & 67,54 \\
\hline Österreichisch-Ungarische Bank . & 77,53 & 82,20 & 79,02 & 72,67 & 67, or \\
\hline Banca d'Italia . . . . . . . & 75,36 & 76,86 & 73,76 & $7 \mathrm{I}, 82$ & 69,85 \\
\hline Ryska Riksbanken . . . . . & 98,99 & IO7,34 & $109,5 \mathrm{I}$ & 105,09 & 99,36 \\
\hline Finlands Bank. & 28,03 & 29,83 & 22,16 & $22,8 \mathrm{I}$ & 28,38 \\
\hline Sveriges Riksbank . . . & 40,44 & 43,85 & 43,79 & 43,42 & $46,2 \mathrm{I}$ \\
\hline Norges Bank . . . . & 37,42 & $39, \mathrm{r} 8$ & 39,03 & $4 \mathrm{I}, \mathrm{I} 9$ & 39,50 \\
\hline Nationalbanken i Köpenhamn & 60,00 & $6 \mathrm{I}, 85$ & 57,05 & 55,66 & 53,57 \\
\hline De Nederlandsche Bank ... & 53,49 & 57,69 & 51,19 & 54,54 & $52,8 \mathrm{I}$ \\
\hline Banque Nationale de Belgique . & 20,18 & 20,63 & $2 \mathrm{I}, 74$ & 27,57 & 28,03 \\
\hline Schweizerische Nationalbank . . & 72,95 & $7 \mathrm{I}, 4 \mathrm{I}$ & 66,03 & 68,35 & 67,25 \\
\hline Banque Nationale privilégiée du & & & & & \\
\hline Royaume de Serbie..... & 49,38 & 42,06 & 56,18 & 58,62 & 67,24 \\
\hline Banque Nationale de Bulgarie . & 65,20 & 53,12 & 68,62 & 56,85 & 48,04 \\
\hline Banque Nationale de Roumanie. . & 35,19 & 33,90 & 34,52 & 35,66 & 34,08 \\
\hline Banco de España . . & - & 71,56 & 69,25 & 68,27 & 64,75 \\
\hline Banco de Portugal . . . . & I 3,59 & 16,62 & 16,77 & 15,36 & I $8, \mathbf{I}_{4}$ \\
\hline
\end{tabular}




\section{Sedelcirkulationens täckning af guld i procent.}

\begin{tabular}{|c|c|c|c|c|c|}
\hline & 1908 & 1909 & 1910 & I9II & 1912 \\
\hline Bank of England $\ldots \ldots$ & 126,34 & 125,33 & I $27,9 \mathrm{I}$ & I 30,60 & I $3 \mathrm{I}, 78$ \\
\hline Banque de France . . . . . & 62,88 & $7 \mathrm{I}, 47$ & $65,4 \mathrm{I}$ & $6 I, 14$ & 60,85 \\
\hline Reichsbank . . . . . . & $5 \mathrm{I}, 52$ & 50,44 & 48,44 & 49,75 & 49,39 \\
\hline Österreichisch-Ungarische Bank .. & 60,96 & $66,9 \mathbf{r}$ & 64,22 & 59,32 & $54,5^{8}$ \\
\hline Banca d'Italia . . . . . . & 66,96 & 68,52 & 66,89 & 65,39 & 62,68 \\
\hline Ryska Riksbanken . . • • • • & 92,59 & 100,27 & IO3,OI & 99,58 & 94,12 \\
\hline Finland Bank . . . . . • & $25,7 \mathrm{I}$ & 26,83 & 19,93 & 20,94 & 26,43 \\
\hline Sveriges Riksbank . . . . & 40,44 & 43,85 & 43,79 & 43,42 & $46,2 \mathrm{I}$ \\
\hline Norges Bank . . . • • • • & 37,42 & 39,18 & 39,03 & $4 \mathrm{I}, 19$ & 39,50 \\
\hline Nationalbanken i Köpenhamn . • & - & 56,67 & $52, \mathrm{II}$ & 50,98 & 49,12 \\
\hline De Nederlandsche Bank . . • & 34,74 & 42,63 & $4 \mathrm{I}, \mathrm{I6}$ & 47,12 & 49,34 \\
\hline Banque Nationale de Belgique .. & 一 & - & 一 & $19,9 x$ & 20,95 \\
\hline Schweizerische Nationalbank . . & $67,4 \mathrm{I}$ & 63,47 & 59,38 & $62,5 \mathrm{I}$ & $62,4 \mathrm{I}$ \\
\hline \multicolumn{6}{|l|}{ Banque Nationale privilégiée du } \\
\hline Royaume de Serbie . • • & 30,80 & 28,37 & 42,66 & 47,01 & 58,96 \\
\hline Banque Nationale de Bulgarie . & 38,36 & 32,50 & 43,10 & 35,72 & $33,9 \mathrm{r}$ \\
\hline Banque Nationale de Roumanie . & 34,75 & 33,58 & 34,18 & 35,43 & 33,92 \\
\hline Banco de España $\cdot \cdots \cdot \cdots$ & - & 23,88 & 23,87 & 23,88 & $23,4^{8}$ \\
\hline Banco de Portugal . & 7,44 & 7,88 & 8,43 & 8,23 & 8,27 \\
\hline
\end{tabular}

\section{Samtliga avistaförbindelsernas täckning af metall i procent.}

\begin{tabular}{|c|c|c|c|c|c|}
\hline & I 908 & 1909 & I9Io & I9II & I912 \\
\hline Bank of England...... & 45,39 & 44,79 & 44,44 & $45,3^{8}$ & 43,82 \\
\hline Banque de France $\ldots . .$. & 70,87 & 75,78 & 71,30 & 66,47 & 64,19 \\
\hline Reichsbank . . . . . . & 46,89 & $45,5 \mathrm{r}$ & 46,83 & 48,74 & 48,14 \\
\hline Österreichisch-Ungarische Bank . . & 70,92 & 74,93 & $7 \mathrm{I}, 74$ & 66,20 & 60,69 \\
\hline Banca d'Italia . . . . . • & 68,82 & $70, \mathbf{1 3}$ & 67,82 & 66,15 & $64,4 \mathrm{I}$ \\
\hline Ryska Riksbanken . . . . . & $64, \infty$ & 67,42 & 66,40 & 59,90 & $56,7 \mathrm{x}$ \\
\hline Finlands Bank . . . . • • • & 21,88 & 24,55 & 19,22 & I 8,78 & 22,94 \\
\hline Sveriges Riksbank . . . . . & 32,36 & 35,70 & 35,15 & 35,06 & 36,76 \\
\hline Norges Bank . • • • • • • & 33,93 & 35,79 & 35,84 & 37,86 & 36,40 \\
\hline Nationalbanken i Köpenhamn $\cdot$. & 58,02 & 59,49 & $54,5 x$ & 53,42 & 51,62 \\
\hline De Nederlandsche Bank . . • & 52,23 & 56,22 & 50,04 & 52,96 & $5 \mathrm{I}, 47$ \\
\hline Banque Nationale de Belgique . . & I8,19 & 18,59 & 19,79 & 24,60 & 25,39 \\
\hline Schweizerische Nationalbank . • & 62,13 & 59,85 & $52,9 \mathrm{r}$ & 55,50 & 57,97 \\
\hline \multicolumn{6}{|l|}{ Banque Nationale privilégiée du Roy- } \\
\hline aume de Serbie $\ldots \cdot \cdots$ & 47,60 & 38,95 & 52,77 & 55,50 & 62,57 \\
\hline Banque Nationale de Bulgarie .. & - & 40,67 & 56,37 & 50,76 & - \\
\hline
\end{tabular}


Sanıtliga avistaförbindelsernas täckning af guld i procent.

\begin{tabular}{|c|c|c|c|c|c|}
\hline & 1908 & 1909 & I9IO & I9II & I912 \\
\hline Bank of England $\ldots \ldots$ & $44,4^{2}$ & 43,84 & 43,48 & 44,43 & 42,92 \\
\hline Banque de France . . . . . . & 54,67 & $60,8 \mathrm{r}$ & 56,89 & 52,79 & $5^{1, f_{2}}$ \\
\hline Reichsbank . . . . . . & $36, \mathbf{1 3}$ & 34,59 & 34,50 & 35,72 & 35,20 \\
\hline Österreichisch-Ungarische Bank . . & 55,76 & 60,99 & 58,30 & 54,03 & 49,43 \\
\hline Banca d'Italia . . . . . . & $6 \mathrm{I}, \mathbf{2 x}$ & 62,53 & $6 \mathrm{I}, 50$ & 60,22 & $57,8 \mathrm{c}$ \\
\hline Ryska Riksbanken . . . . . & 59,85 & 62,98 & 62,45 & 56,76 & $53,7^{2}$ \\
\hline Finlands Bank . . . . . . . & 20,07 & 22,09 & $\mathrm{I} 7,28$ & 17,24 & $2 \mathrm{I}, 36$ \\
\hline Sveriges Riksbank . . . . . . & 32,36 & 35,70 & 35,15 & 35,06 & 36,76 \\
\hline Norges Bank . . . . . . & 33,93 & 35,79 & 35,84 & 37,86 & 36,40 \\
\hline Nationalbanken i Köpenhamn . . & - & $54,5 \mathrm{r}$ & 49,79 & 48,93 & $47,3^{2}$ \\
\hline De Nederlandsche Bank . . . & 33,92 & $4 \mathrm{I}, 54$ & 40,23 & 45,75 & 48,08 \\
\hline Banque Nationale de Belgique . . & - & - & - & 17,76 & 18,97 \\
\hline Schweizerische Nationalbank . . . & $57,4 \mathrm{I}$ & 53,19 & 47,57 & 50,77 & 53,79 \\
\hline \multicolumn{6}{|l|}{ Banque Nationale privilégiée du Roy- } \\
\hline aume de Serbie...$\cdot$ & 29,69 & 26,28 & 40,07 & 44,50 & 54,87 \\
\hline Banque Nationale de Bulgarie . . & - & 24,88 & $35,4 \mathrm{I}$ & $3^{\mathrm{I}, 90}$ & - \\
\hline
\end{tabular}

\section{Växeldiskonto.}

\begin{tabular}{|c|c|c|c|c|c|}
\hline & 1908 & 1909 & I910 & I9II & I9I 2 \\
\hline Bank of England . . . . . & 3, or & 3,10 & 3,72 & 3,47 & 3,77 \\
\hline Banque de France $\ldots \ldots$ & 3,04 & $3, \infty$ & $3, \infty$ & $3, \mathbf{1 3}$ & 3,37 \\
\hline 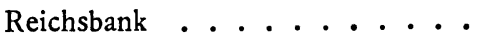 & 4,76 & 3,93 & 4,35 & 4,40 & 4,95 \\
\hline Österreichisch-Ungarische Bank . • & 4,25 & $4, \infty$ & 4,19 & 4,40 & 5,15 \\
\hline Banca d'Italia ....... & 4,50 & 4,34 & $4,6 x$ & 4,94 & 5,47 \\
\hline Ryska Riksbanken ...... & 5,95 & 4,99 & 4,50 & 4,50 & $5, \infty$ \\
\hline Finlands Bank . . . . . . & $6, \mathbf{1 6}$ & $5, \mathrm{r} 6$ & 5,02 & 4,76 & $5, \mathrm{rc}$ \\
\hline Sveriges Riksbank ...... & 5,88 & 4,69 & 4,63 & 4,57 & $4,8 \mathrm{r}$ \\
\hline Norges Bank . . . . . . . & 5,34 & 4,54 & 4,50 & 4,63 & $5,3^{\varepsilon}$ \\
\hline Nationalbanken i Köpenhamn . . & $6, \mathbf{r}_{4}$ & 4,94 & $5, \infty$ & 4,62 & 5,06 \\
\hline De Nederlandsche Bank . . . & 3,38 & 2,88 & 4,23 & 3,45 & $4, \infty 0$ \\
\hline Banque Nationale de Belgique... & 3,56 & $3, \mathbf{r r}$ & $4^{, 12}$ & 4,15 & 4,49 \\
\hline Schweizerische Nationalbank . • & 3,73 & 3,22 & $3,5 \mathrm{r}$ & 3,70 & 4,20 \\
\hline Banque Nationale de Roumanie. . & 5,18 & $5, \infty 0$ & $5, \infty$ & 5,00 & 5,20 \\
\hline Banco de España . . . . . . & - & 4,50 & 4,50 & 4,50 & 4,50 \\
\hline Banco de Portugal ...... & 5,98 & $6, \infty$ & $6, \infty$ & $6, \infty$ & $6, \infty$ \\
\hline
\end{tabular}


Årsvinstens fördelning mellan staten och aktieăgarna (i procent af ärsvinstens belopp),

\begin{tabular}{|c|c|c|c|c|c|c|c|c|c|c|}
\hline & 19 & 08 & I9 & & 19 & IO & I9 & I I & & 12 \\
\hline & $\begin{array}{c}\text { Sta- } \\
\text { ten }\end{array}$ & $\begin{array}{c}\text { Akt.- } \\
\text { âg. }\end{array}$ & $\begin{array}{l}\text { Sta- } \\
\text { ten }\end{array}$ & $\begin{array}{c}\text { Akt.. } \\
\text { ag.. }\end{array}$ & $\begin{array}{c}\text { Sta- } \\
\text { ten }\end{array}$ & $\begin{array}{c}\text { Akt.- } \\
\text { ag. }\end{array}$ & $\begin{array}{l}\text { Sta- } \\
\text { ten }\end{array}$ & $\begin{array}{c}\text { Akt.. } \\
\text { äg. }\end{array}$ & $\begin{array}{l}\text { Sta- } \\
\text { ten }\end{array}$ & $\begin{array}{c}\text { Akt.- } \\
\text { äg. }\end{array}$ \\
\hline $\begin{array}{l}\text { Reichsbank . • • • • • } \\
\text { Österreichisch-Ungarische }\end{array}$ & 62,2 & 37,8 & 54,5 & 45,5 & 58,0 & 42,0 & $54, \circ$ & 38,3 & 58,2 & 33,5 \\
\hline Bank . . • • • • • & 29,4 & 63,2 & 22,3 & 71,6 & 28,7 & 64,0 & 38,3 & $53, x$ & 45,6 & 44,9 \\
\hline Banca d'Italia.$\cdots \cdot$ & - & - & I8,o & 82,0 & 20,4 & 79,6 & 22,4 & 77,6 & 23,3 & 76,7 \\
\hline Norges Bank . . . . & 25,0 & 75,0 & 25,0 & 75,0 & 25,0 & 75,0 & 28,6 & $7 \mathrm{I}, 4$ & 37,3 & 58,9 \\
\hline Nationalbanken iKöpenhamn ${ }^{1}$ & - & 一 & 30,7 & 69,3 & 31,6 & 68,4 & $3 \mathrm{I}, 6$ & 68,4 & 30,8 & 60,2 \\
\hline De Nederlandsche Bank ${ }^{2}$. & 53,7 & 43,8 & 52,7 & 44,9 & 56,4 & 39,9 & 53,5 & 40,0 & 55,4 & $3^{8,5}$ \\
\hline Banque Nationale de Belgique & 21,0 & 66,2 & $2 I, 0$ & 66,2 & 21,0 & 66,2 & 21,0 & 66,2 & $2 I, I$ & $66, \mathbf{x}$ \\
\hline Schweizerische Nationalbank & 0,8 & 89,2 & 14,0 & 76,0 & 50,4 & 39,6 & 51,2 & 38,8 & 57,0 & 33,0 \\
\hline $\begin{array}{l}\text { Banco de Portugal . . . } \\
\text { Banque Nationale privilégiée }\end{array}$ & I8,3 & $6 \mathrm{I}, 4$ & I 8,7 & 60,9 & 18,7 & 60,9 & 20,6 & 62,5 & $20, \mathbf{x}$ & $64, x$ \\
\hline $\begin{array}{l}\text { du Royaume de Serbie } \\
\text { Inque Nationale de Rou- }\end{array}$ & 14,2 & 70,0 & I4, 0 & 70,5 & $\mathrm{I} 2,3$ & 74,0 & I 2,6 & 73,4 & - & - \\
\hline
\end{tabular}

\section{Utdelningen till aktieägarna i procent af inbetald grundfond.}

\begin{tabular}{|c|c|c|c|c|c|}
\hline & 1908 & 1909 & 1910 & I9II & I9I 2 \\
\hline Bank of England $\ldots \ldots$ & 9,0 & 9,0 & 9,0 & 9,o & 9,0 \\
\hline Banque de France $\ldots \ldots$ & I6,0 & 14,0 & 14,0 & 14,0 & I6,o \\
\hline Reichsbank . . . . . . . & 7,77 & 5,83 & 6,48 & 5,86 & 6,95 \\
\hline Österreichisch-Ungarische Bank . . . & $6,5 \mathrm{I}$ & $5,8 \mathrm{I}$ & 6,45 & 7,46 & 8,57 \\
\hline Banca d'Italia . . . . . . . . & 6,33 & 6,83 & 7,17 & 7,50 & 7,67 \\
\hline Norges bank . . . . . . . & 9,0 & 9,0 & 9,0 & Io,o & IO,o \\
\hline Nationalbanken i Köpenhamn ${ }^{1}$. . . . & $8, \circ$ & 7,0 & 6,5 & 7,0 & 7,0 \\
\hline De Nederlandsche Bank ${ }^{2} \ldots \ldots$ & 8,8 & 8,3 & I I, 7 & 10,2 & $\mathrm{I} 2,2$ \\
\hline Banque Nationale de Belgique . . . & I6,6 & 16,6 & 16,6 & I 6,6 & 16,8 \\
\hline Schweizerische Nationalbank . . . . & 4,0 & 4,0 & 4,0 & 4,0 & 4,0 \\
\hline Banco de España . . . . . . & 19,5 & I9,o & 19,0 & I9, & I9,0 \\
\hline Banco de Portugal . . . . . . & I0,0 & IO,o & Io,o & I 0,0 & Io,o \\
\hline Banque Nationale de Grèce . . . . & - & - & 20,0 & 20,0 & 20,0 \\
\hline \multicolumn{6}{|l|}{ Banque Nationale privilégiée du Royaume } \\
\hline de Serbie $\cdot \ldots \cdot \cdots \cdot \cdot \cdot$ & 9,53 & 10,0 & IO,o & I0,0 & - \\
\hline Banque Nationale de Roumanie . . & 32,4 & 32,8 & $34, \circ$ & 35,2 & $3^{8,8}$ \\
\hline
\end{tabular}

1 Uppgifterna afse räkenskapsåren 1907/8-I9II/ı 2.

$1908 / 9-1912 / 13$. 